\title{
Innovationen ausgezeichnet
}

Im Rahmen einer Gala in Berlin ist der von Springer Medizin gestiftete Galenusvon-Pergamon-Preis für pharmazeutische Innovationen verliehen worden. Mit dem CharityAward wurde auch ehrenamtliches Engagement gewürdigt.

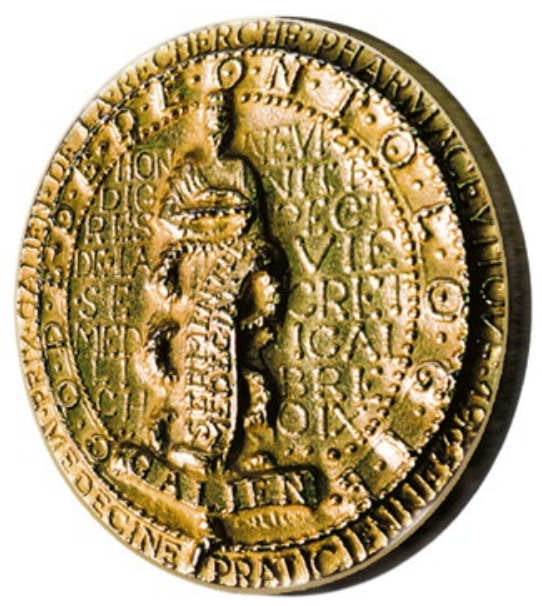

Die Preisträger wurden am 20. Oktober von Bundesgesundheitsminister Hermann Gröhe und dem Jury-Vorsitzenden Professor Erland Erdmann bei der „Springer Medizin Gala“ in Berlin geehrt. Galenus-Preise wurden in vier Kategorien vergeben.

\section{Grundlagenforschung}

Den Preis in der Kategorie "Grundlagenforschung" erhielt Dr. Michael Potente vom Max-Planck-Institut für Herz- und Lungenforschung, Bad Nauheim. Potente und seine Arbeitsgruppe belegten, dass der Verlust des Transkriptionsfaktors FOXO1 zu einem unkontrollierten Wachstum des Endothels und einer Vergrößerung von Blutgefäßen führt. Hingegen bedeutet ein Funktionsgewinn die Ausdünnung des Endothels und ein verringertes Verzweigungsvermögen von Blutgefäßen. Potentes Forschung könnte zum Dreh- und Angelpunkt für zukünftige therapeutische Ansätze werden.

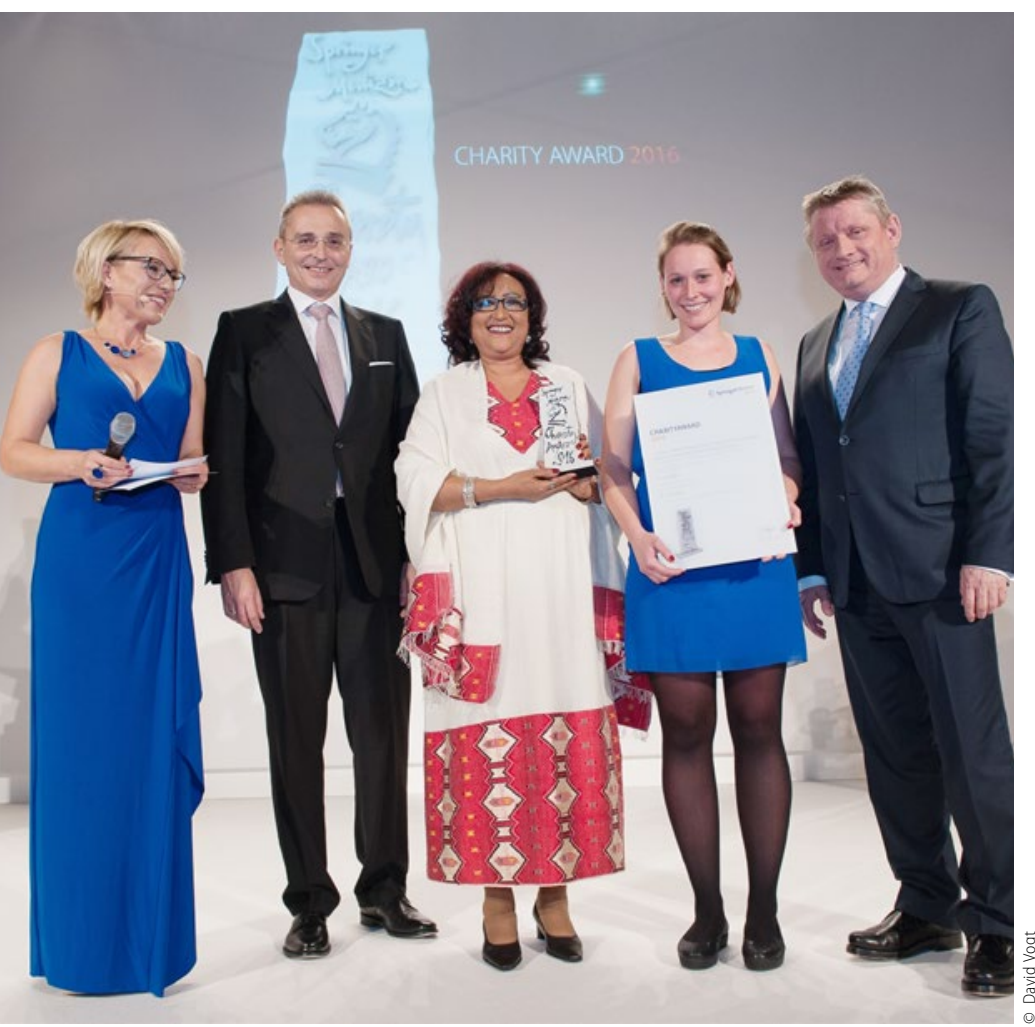

Gewinnerinnen des CharityAwards: Shewa Sinn (mit Trophäe) und Julia Schwieterjann (mit Urkunde) von AGISRA, im Bild mit (v. l.) Moderatorin Andrea Ballschuh, Verlagschef Joachim Krieger vom Stifter und Sponsor Springer Medizin und Minister Hermann Gröhe.

\section{Primary Care}

In der Kategorie „Primary Care“ ging der Preis an Empagliflozin (Jardiance ${ }^{\star}$ ) von Boehringer Ingelheim, das Typ-II-Diabetikern verordnet wird. Das Präparat verbessert aufgrund seines Wirkmechanismus die Blutzuckerkontrolle dieser Patienten. Zudem konnte für den Wirkstoff eine erhebliche Reduktion des kardiovaskulären Risikos bei entsprechender Erkrankung belegt werden.

\section{Specialist Care}

Den Preis in der Kategorie „Specialist Care" hat die Jury in diesem Jahr an zwei Innovationen aus der Immunonkologie vergeben: Nivolumab (Opdivo ${ }^{\circ}$ von Bristol-Myers Squibb sowie Pembrolizumab (Keytruda ${ }^{\circ}$ ) von MSD Sharp \& Dohme. Beide Antikörper gehören zur neuen Wirkstoffklasse der PD1 (Programmed Death 1)-Hemmer. Diese Immun-Checkpoint-Hemmer versetzen das körpereigene Immunsystem in die Lage, Tumoren eigenständig wirksam zu bekämpfen. Pembrolizumab hat die Therapie von Patienten mit fortgeschrittenem, nicht resezierbarem oder metastasiertem Melanom vorangebracht, Nivolumab darüber hinaus von Patienten mit lokal fortgeschrittenem oder metastasiertem nicht kleinzelligem Lungenkarzinom und von Patienten mit fortgeschrittenem Nierenzellkarzinom. Ein therapeutisches Potenzial wird auch außerhalb der Immunonkologie vermutet.

\section{Orphan Drugs}

In der Kategorie „Orphan Drugs“ ging der Preis an Sebelipase alfa $\left(K_{\text {Kanuma }}{ }^{\circ}\right.$ ) von Alexion. Dabei handelt es sich um die erste Enzymersatztherapie für Patienten mit LAL-Defizienz, eine genetisch bedingte, seltene Stoffwechselerkrankung bei der sich Cholesterinester und Triglyzeride vermehrt in verschiedenen Geweben ansammeln und dort multiple Organschäden verursachen, die zum vorzeitigen Tod führen können.

red

\section{CharityAward 2016}

Bereits zum achten Mal in Folge würdigt Springer Medizin ehrenamtliches Engagement. Die drei Preisträger erhalten jeweils eine Kombination aus Preisgeld und Medienleistungen.

Platz 1 ging an AGISRA, eine Informations- und Beratungsstelle in Köln, die sich seit 1993 für Menschenrechte und Interessen von Migrantinnen und Flüchtlingsfrauen einsetzt.

Platz 2 belegte die "Ambulante Sozialpädagogik Charlottenburg" (AMSOC) in Berlin, bei der seit 2005 Paten die Verantwortung für Kinder psychisch erkrankter Eltern übernehmen.

Mit dem 3. Platz ausgezeichnet wurde die "Sprechstunde für Menschen ohne Sozialversicherung" von Dr. Klaus Harbig in Dortmund. 